\title{
Enhanced Power and Ground Mesh Structure for IR-Drop Reduction
}

\author{
Vakhtang Janpoladov1, Suren Abazyan² \\ ${ }^{1}$ Russian-Armenian University, Yerevan, Armenia \\ ${ }^{2}$ Yerevan State University, Yerevan, Armenia \\ Email: su.abazyan@gmail.com
}

How to cite this paper: Janpoladov, V. and Abazyan, S. (2020) Enhanced Power and Ground Mesh Structure for IR-Drop Reduction. Open Access Library Journal, 7: e6334.

https://doi.org/10.4236/oalib.1106334

Received: April 18, 2020

Accepted: May 10, 2020

Published: May 13, 2020

Copyright $\odot 2020$ by author(s) and Open Access Library Inc.

This work is licensed under the Creative Commons Attribution International License (CC BY 4.0).

http://creativecommons.org/licenses/by/4.0/

(c) (i) Open Access

\begin{abstract}
New enhanced power/ground mesh structure is presented for voltage drop (IR drop) reduction on power distribution network in integrated circuits (ICs). The new structure focuses on the length of the overall power/ground mesh wire pattern, reducing it by using a complex dotted line wire pattern instead of the usual mesh straps, which brings to wire length reduction on almost $40 \%$, while via count on changed metal layer remained the same. Measurements of the voltage drop prove that with proposed mesh structure IR drop reduced about $76 \%$ for power net compared to standard mesh structure but runtime for mesh creation is increased due to more complex patterns. Proposed structure can be used in advanced technology nodes where IR drop phenomenon has become critical for performance of the ICs.
\end{abstract}

\section{Subject Areas \\ Computer Engineering}

\section{Keywords}

IR Drop, Power and Ground Mesh, Wire Length, Resistance, Measurement

\section{Introduction}

The current pace of technology progress in the field of ICs brings new challenges in design and verification processes. With the shrink of technology node, in addition to the appearance of new problems, the old, already well-known phenomena, are beginning to have more and more significance in correct IC operation. In this paper, the phenomenon of voltage drop is considered. Voltage drop called IR drop is a process, when voltage from source moves to the load through mesh, created from metal wires, with some losses. IR drop impacts on circuit 
performance, which is a major parameter in IC market today. Customers have also tightened IR drop requirements to limit a drop of no more than $1 \%-4 \%$ of the supply voltage.

Many works are focusing on IR drop problem and ways to perform fast analyzes and prediction of drop value [1] [2] [3], but this work is focused on changing power grid to improve IR drop. Papers [4] [5] are focused on placement and post-routing optimization for IR drop reduction. The work [6] describes diagonal routing in power/ground mesh, but nowadays IC design engineers commonly use orthogonal power/ground net routing. IR drop value depends on overall resistance of the power distribution network and flowing current through it. Resistance depends linearly on wire length, so we created new power/ground mesh pattern with orthogonal routing and reduced wire length. The other goal of this study is not to change the connections (vias) count of power/ground mesh.

\section{Standard Structure Implementation}

For this study, test case was created. First iteration, as described above, is done with commonly used mesh structure shown in Figure 1, which is created with Metal 5 and Metal 6 metal layers. Orthogonal structure is used in order to reduce parasitic cap between metal wires, which could lead to bigger voltage drop values. Vertical and horizontal straps are connected to the power ring of the chip. Metal 6 is connected to Metal 5 through vias. Metal 1 - Metal 4 layers are used for signal and clock routing.

Main parameters for mesh creation are metal width (w), spacing (s) and pitch (p). Wire pitch parameter shows the distance between the axes of the two metal wires. Spacing is a distance between the edges of the two wires.

Standard mesh created with parameters described in Table 1.

After standard mesh implementation results were collected which are necessary for comparison with newly proposed method. Their values are demonstrated in Table 2.

\section{Proposed Structure}

A novel method of power and ground mesh structure is proposed and shown in Figure 2 .

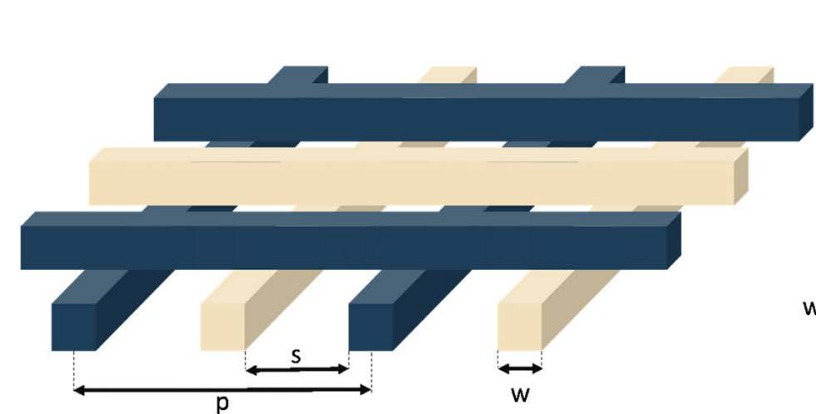

(a)

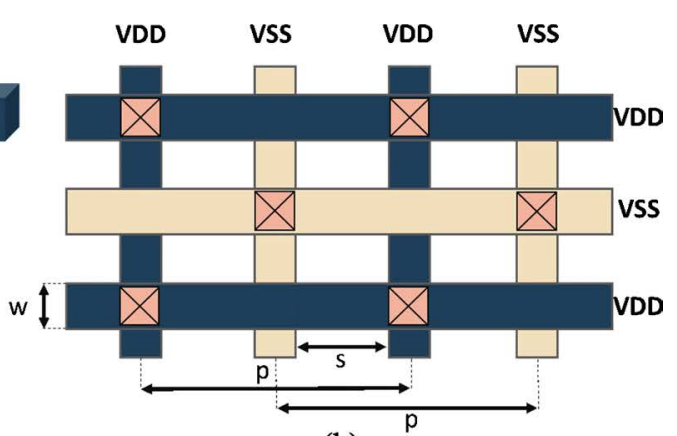

(b)

Figure 1. Standard orthogonal structure. (a) 3d view; (b) Top view. 
Table 1. Mesh wiring parameters.

\begin{tabular}{ccc}
\hline & \multicolumn{2}{c}{ Layer } \\
\cline { 2 - 3 } Parameter & Metal 5 & Metal 6 \\
\hline Pitch (p) & 9 um & 9 um \\
Spasing (s) & $4.5 \mathrm{um}$ & $4.5 \mathrm{um}$ \\
Width (w) & $2 \mathrm{um}$ & $2 \mathrm{um}$ \\
\hline
\end{tabular}

Table 2. Standard mesh reports.

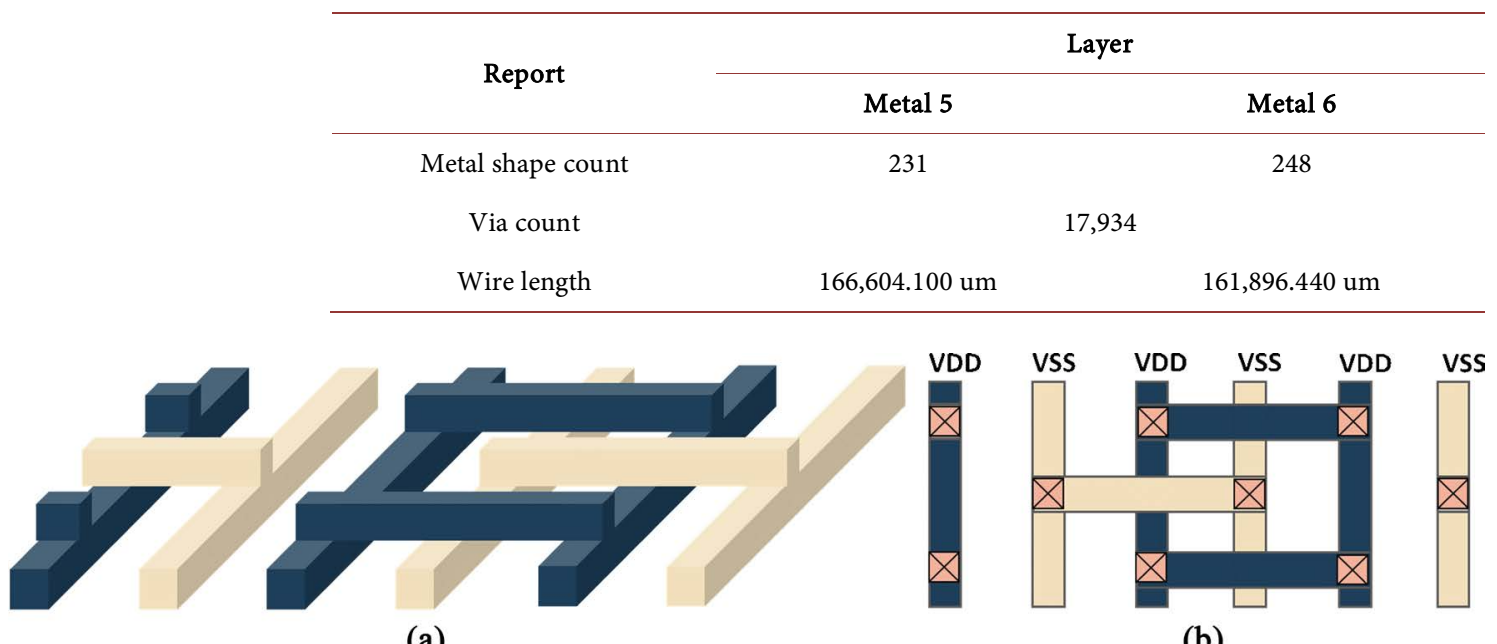

(a)

(b)

Figure 2. Proposed structure. (a) 3d view; (b) Top view.

The idea is to trim one of the upper layer metals between every second pair of power and ground nets, so it will look like a dashed line both for power and ground buses. Described action will lead to wire length reduction. IR drop value depends on resistance of wire mesh, so by reducing its total wire length we can reduce total resistance. Another advantage of proposed structure is keeping via count the same in mesh, so there is no loss in connection count.

Experimental results demonstrated in below chapter prove that created structure reduces length of wires in power distribution network, which brings to IR drop reduction.

\section{Experimental Results}

As described above, new mesh structure was implemented, and general parameters (pitch, width, spasing) were kept the same for similar IR drop analyzes. After the implementation routing reports were collected for analyzes and comparison in Table 3 and Table 4.

Metal shapes count significantly increased on layer Metal 6 due to cut of usual straps. Comparison of routing reports shows, that total wire length on Metal 5 and Metal 6 layers decreased by almost $40 \%$. Via count remained the same.

To prove the effectiveness of proposed power and ground mesh structure IR drop analyzes were done. Results were collected for comparison with standard 
Table 3. Standard mesh reports.

\begin{tabular}{ccc}
\hline \multirow{2}{*}{ Report } & \multicolumn{2}{c}{ Layer } \\
\cline { 2 - 3 } & Metal 5 & Metal 6 \\
\hline Metal shape count & 231 & 248 \\
Via count & & 17,934 \\
Wire length & $166,604.100$ um & $166,604.100$ um \\
\hline
\end{tabular}

Table 4. Standard mesh reports.

\begin{tabular}{cccc}
\hline Report & Layer name & Standard structure & Proposed structure \\
\hline \multirow{2}{*}{ Wire length } & Metal 5 & $166,604.100 \mathrm{um}$ & $166,604.100 \mathrm{um}$ \\
Via count & Metal 6 & $161,896.440 \mathrm{um}$ & $98,815.120 \mathrm{um}$ \\
\hline
\end{tabular}

Table 5. IR drop comparison.

\begin{tabular}{cccc}
\hline Supply net & Ideal voltage [v] & $\begin{array}{c}\text { Standard mesh drop } \\
\text { voltage }[\mathbf{v}]\end{array}$ & $\begin{array}{c}\text { Proposed mesh drop } \\
\text { voltage }[\mathbf{v}]\end{array}$ \\
\hline VDD (Power) & 0.99 & $6.7 \mathrm{e}-05$ & $1.6 \mathrm{e}-05$ \\
VSS (Ground) & 0 & $7.8 \mathrm{e}-05$ & $3.2 \mathrm{e}-05$ \\
\hline
\end{tabular}

structure to show the improvement. For this test, supply voltage is $0.99 \mathrm{~V}$ and test temperature is $125^{\circ} \mathrm{C}$ with fast-fast transistor technology process. Power and ground wires are named VDD and VSS correspondingly in test design. RedHawk tool for IR drop value calculation used to test the effectiveness of proposed structure.

For correct understanding of the changes, a numerical analysis of both standard and new mesh structures was performed, which is shown in the Table 5.

Based on tool calculation we can prove, that proposed structure brings huge reduction of IR drop.

\section{Conclusion}

Observed experimental results proved the effectiveness of proposed power and ground mesh structure. Worst value decreased about $76 \%$ on power net and about $60 \%$ on ground net based on tool calculations, without loss of any via connection with expected losses in runtime, due to more complex mesh architecture. Proposed mesh can be used in designs where influence of IR drop phenomenon is critical.

\section{Conflicts of Interest}

The authors declare no conflicts of interest regarding the publication of this paper.

\section{References}

[1] Kose, S. and Friedman, E.G. (2012) Efficient Algorithms for Fast IR Drop Analysis 
Exploiting Locality. Integration, 45, 149-161.

https://doi.org/10.1016/j.vlsi.2011.09.003

[2] Abazyan, S.S. and Mamikonyan, N.E. (2020) Static IR Drop Estimation on the Power Network. Open Access Library Journal, 1, 1-7.

https://doi.org/10.4236/oalib.1105995

[3] Kahng, A.B., Liu, B. and Wang, Q. (2007) Stochastic Power/Ground Supply Voltage Prediction and Optimization via Analytical Placement. IEEE Transactions on Very Large Scale Integration (VLSI) Circuits, 15, 904-912. https://doi.org/10.1109/TVLSI.2007.900745

[4] Atulya Ratna, G. and Padma Priya, K. (2016) A Post-Routing Stage IR Drop Reduction Technique with Less Routing Resources. IEEE International Conference on Computational Intelligence and Computing Research, Chennai, 15-17 December 2016, 1-6. https://doi.org/10.1109/ICCIC.2016.7919634

[5] Heo, S.I., Kahng, A.B., Kim, M., Wang, L. and Yang, C. (2019) Detailed Placement for IR Drop Mitigation by Power Staple Insertion in Sub-10nm VLSI. 2019 Design, Automation \& Test in Europe Conference \& Exhibition (DATE), Florence, 25-29 March 2019, 830-835. https://doi.org/10.23919/DATE.2019.8715096

[6] Rao, L.D. and Sivasankaran, K. (2018) Power Grid Strategies for Minimum IR Drop in Multi Power Domain Design. 2018 3rd IEEE International Conference on Recent Trends in Electronics, Information \& Communication Technology (RTEICT), Bangalore, 18-19 May 2018, 1196-1201. https://doi.org/10.1109/RTEICT42901.2018.9012645 